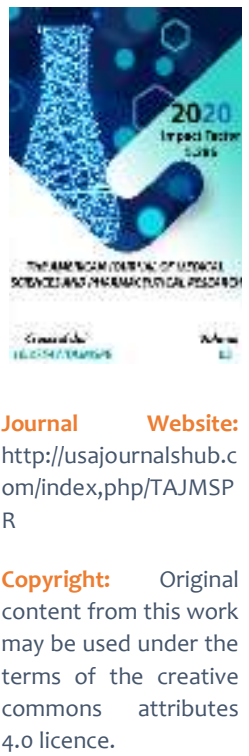

\title{
Clinical And Laboratory Results Of Intrauterine Device Cu T 380 A Intrauterine System Intraoperative Injection
}

\author{
Nasirova Zebiniso Azizovna \\ Samarkand State Medical Institute, Samarkand, Uzbekistan \\ Negmadjanov Bahodur Boltaevich \\ Samarkand State Medical Institute, Samarkand, Uzbekistan
}

\section{ABSTRACT}

Effective and safe contraceptive methods not only prevent unplanned pregnancies and their termination, but also make it possible to fully restore the mother's body after surgery to carry out the necessary pre-pregnancy preparation. The goal of the study was to evaluate the clinical outcome of postplacental administration of $\mathrm{Cu}$ T $380 \mathrm{~A}$ in women during a caesarean section. The study was conducted at Samarkand Maternity Hospital No. 2 to assess the clinical outcome of postplacental administration of Cu T $380 \mathrm{~A}$ in women during caesarean section. The present study showed that there were no serious complications and only minor side effects such as pain, fever, bleeding and irregular bleeding, which were observed only in some women during the hospital stay and the control visit in the first 6 months. The determination of vaginal BMW tendrils was made possible in $72.12 \%$ of women during the control visit after 6 weeks. The visibility of the tendrils increased over time and after 6 months they were seen in $90.81 \%$ of cases. Exposure frequency was 2 cases ( $4 \%$ ) and another 2 women (4\%) required removal of the PMTCT for various reasons, resulting in a $92.3 \%$ retention rate six months after contraception. No cases of pregnancy were registered. Post-placental introduction of BIC Cu T 380 A during a C-section is a practical, convenient, safe, effective and acceptable contraceptive method.

\section{KEYWORDS}

Clinical results, BIC Cu T 380 A, family planning, postpartum contraception. 


\section{INTRODUCTION}

The development and improvement of obstetrical science and modern technology in many areas of medicine has led to a significant increase in the indications for delivery through Caesarean section surgery. This has led to the fact that Caesarean section is currently the most common delivery procedure. In Uzbekistan, the frequency of this operation has more than doubled over the past 10-15 years, and according to the Statotadel of RSNPC A and G and ROC, it was $18 \%$ in 2017, reaching $35-40 \%$ in some institutions [2].

The problem of contraception after a C-section is very important, both in medical and social terms. It has been studied that in the interval between prompt delivery and subsequent pregnancy of more than 2 years, maternal mortality is reduced by $32 \%$ and infant mortality by $10 \%$. Conception after caesarean section reduces the frequency of medical abortions by $90 \%$. The onset of pregnancy within a year after a C-section is accompanied by a significant increase in the risk of gestational complications: spontaneous abortions, premature births, placental insufficiency, uterine rupture, etc. [3].

BIC are among the most commonly used effective and reversible contraceptive methods among women of reproductive age worldwide. Post-placental BMW is an effective and safe contraceptive method. Taking advantage of antenatal care and family planning counseling in the antenatal period at the primary level and in the maternity ward, the introduction of BMI Cu T $380 \mathrm{~A}$ immediately after delivery is a good option as a contraceptive method.
Direct postplacental administration of BIC Cu T 380 A during a Caesarean section provides a good opportunity to achieve long-acting reversible contraception (LARC) with minimal discomfort for women. The effectiveness of introducing LARC during a Caesarean section without any additional risk of infection has also been reported in various studies. This is increasingly practiced after safety has been reported and the frequency of expulsions decreases after intraoperative BIC administration. This method offers the obstetrician-gynecologist the opportunity to introduce $\mathrm{BIC}$ into the uterus under visual control, thus eliminating the fear of uterine perforation during the procedure $[2,3]$.

Starting to use BMW during a Caesarean section offers an additional benefit, as it eliminates the six-week postpartum waiting period and additional visits to a medical facility. However, despite safety and efficiency, obstetrician-gynecologists still hesitate to use $B M B C 380 A$ for women who have undergone surgical delivery.

The introduction of BMB during a Caesarean section may be an alternative to sterilisation for some couples, especially for women who give birth again and for groups of women who refuse sterilisation for religious reasons. However, the safety and acceptability of postplacental BMW during a Caesarean section has not been widely studied in our country. In view of the above, this study is being conducted to determine the safety and efficacy of postplacental $\mathrm{Cu} \quad \mathrm{T} 380 \mathrm{~A}$ postplacental administration during a Caesarean section. 


\section{RESEARCH METHODS}

A prospective study was conducted in Samarkand City Maternity Hospital No. 2 to assess the clinical outcome of postplacental $\mathrm{Cu}$ $\mathrm{T} 380 \mathrm{~A}$ postplacental administration during a caesarean section. The study included 50 women who were allowed to give birth by caesarean section and wanted to use BIC Cu T $380 \mathrm{~A}$, who met WHO standard medical criteria for BIC administration and were ready to follow the study protocol.

The criteria for inclusion were the following:

- Age 18-45 years

- The gestational age is 37-42 weeks.

- Absence of infectious diseases

- Absence of intra-natal bleeding.

- $\mathrm{Hb} \geq 90 \mathrm{hl} / \mathrm{l}$.

The criteria for exclusion were:

- Women who did not meet the WHO medical eligibility criteria for the application of the BMC

- Known allergy to copper.

- Small pelvic inflammatory diseases in the history.

- Prenatal outflow of amniotic water

- Women with uterine abnormalities

- HIV / AIDS 4 stages.

Women were advised about the postplacental introduction of the BMC during antenatal visits and/or after hospitalisation. The women were told in detail about the study, including the advantages and limitations of the different methods, and repeated counselling was provided before the Caesarean section. Written informed consent was obtained from the women, who were willing to participate in the study and comply with the study protocol. The introduction was carried out after removal of the placenta using Kelly pincers / manually through the uterine incision and the device was placed at the bottom of the uterus. No attempts were made to direct the BMC antennae into the vagina. Antibiotics were injected according to the protocol of the Maternity Hospital for Caesarean section. Women were observed daily for postpartum bleeding as well as any other complaints during the whole period of hospitalization. Before discharging the patient, the following were indicated on the exchange card: type of BMI, date of administration and expiry date. Participants were asked to return to scheduled control visits in 6 weeks, 3 months and 6 months. The date of the first control visit was indicated on the discharge form and a reminder was made by telephone.

If there were any complaints, such as:

- Vaginal secretions with an unpleasant odour, different from ordinary dickheads

- Pain in the lower abdomen.

- Fever with chills.

- Suspicion that the BMC has dropped out.

- Bleeding from the genital tract or a feeling of pregnancy

patients were examined before their planned appearance.

\section{RESULTS}

Most women were between 21 and 30 years old (77.89\%). Two women (4\%) were over 36 years old and only one woman was under 20 years old.

Of the 50 women surveyed, 23 (46\%) had higher education and 37 had secondary and specialized secondary education. The acceptability of $\mathrm{Cu}$ T $380 \mathrm{~A}$ increases with the level of education. Most women (96\%) were registered as married.

Obstetric anamnesis of the women surveyed. Eleven women out of 50 (22\%) in the study had 
their first births (abdominal deliveries) and 39 women $(78 \%)$ had their first re-births. Twentysix $(66.7 \%)$ women $(66.7 \%)$ who had had BMW during a Caesarean section had a natural delivery pathway in the past, eight (20.5\%) had a previous Caesarean section and 5 (12.8\%) had two or more previous Caesarean sections.

The majority of women (38 cases) had a Csection between 37-40 weeks of gestation (74\%) and between 40-42 weeks in 12 cases (24\%). None of the patients with a gestation period of $<37$ weeks were included in the study (Table 2). All women with planned C-section were included in the study. Nineteen women (38\%) were consulted about the introduction of BIC during the antenatal C-section. These women were re-consulted before the Caesarean section and written informed consent was obtained. While the remaining 31 women (62\%) had their first consultation at the maternity hospital before the Caesarean section. 39 women were discharged on the 5 th day after the operation (78\%) without serious complications, while the remaining women (22\%) had a stay in hospital of 6-7 days. None of the women needed to stay longer than 7 days.

Side effects after administration and results: Pain during hospital stay was the most frequent complaint since the introduction of the BMW, which was observed in 5 out of 50 women (10\%). The next one was fever, in 3 women (6\%). There were no cases of postpartum bleeding, lochia with unpleasant odour, sepsis, wound infection or complications from urinary tract. The most frequent complaint during the first visit 6 weeks after delivery was pelvic pain (12\%), followed by extensive serous vaginal discharge (6\%) and irregular bleeding (4\%). None of the women reported fever or wound infection (Table 5). On the second control visit 3 months after delivery, pelvic pain was also the main complaint of women (12\%). Other symptoms reported by the women were haemorrhage (6\%) and excessive serous discharge from the vagina (4\%). No cases of fever or wound infection were reported. On the third control visit after 6 months, pain (8\%) was the most frequent complaint, followed by heavy menstrual bleeding (4\%) and heavy discharge (4\%). None of the women had fever or a wound infection.

In our study we did not observe any serious complications with BIC intraoperative administration, only a few patients reported minor side effects such as pain, discharge and irregular bleeding. At the first control visit after 6 weeks, the antennae were visible at $72 \%$ (36/50). However, tendrils were not visible in 14 (28\%) women at the first control visit.

Visibility of CMB tendrils increased in 6 more cases during the second control visit in the 3 rd month, and in 45 out of 50 cases (90\%) tendrils were visible on the third visit (after 6 months). In the remaining 5 cases, the whiskers were not visible even on the third visit. This required an additional ultrasound investigation. At the same time, a bright echo of the copper BMC was detected with pronounced back shading (Fig. 2).

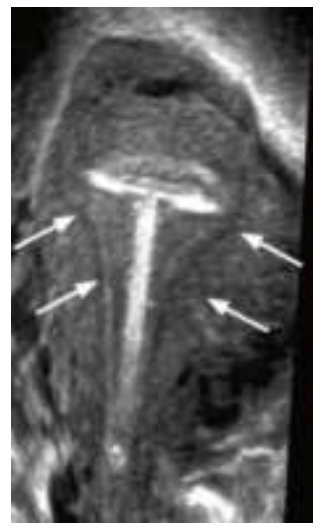

The three-dimensional sonogram shows BMK CIT 380 A correctly positioned in the endometrial cavity. During the first control 
visit after 6 weeks there were no cases of BMK expulsions. During the second control visit in the 3 rd month, there was 1 (2\%) partial BMK expulsions, which necessitated the removal of the BMK. An additional woman then asked for the removal of the $B B Q$ due to the requirement of a sexual partner. During the third control visit after 6 months there was one case of complete BIC CU T $380 \mathrm{~A}$, and in 1 case $\mathrm{Cu}$ T was removed at the request of the woman. Thus, at the end of the study, after 6 months of postnatal care, 2 cases (4\%) of total BMK expulsions and 2 cases ( $4 \%$ ) of removal at the request of the patient were recorded.

\section{DISCUSSION}

Our study found that $77.89 \%$ of patients who received $B M C$ treatment were in the $21-30$ age group. This was probably due to the fact that the majority of patients admitted for delivery were also in the 20-30 age group. It was noted that the acceptability of CMB C T $380 \mathrm{~A}$ increased with an increase in the level of education. This can be explained by the fact that women with education are better informed about modern effective contraceptive methods. This result confirms the importance of education in reducing the risk of unplanned pregnancy. These results are consistent with the Starr K data. A. et al. [11], which showed that education has a positive effect on modern contraceptive use. Nineteen women (38\%) were consulted on the introduction of BMW during the antenatal caesarean section. These women were reconsulted before the Caesarean section and their written informed consent was obtained. While the remaining 31 women (62\%) were counselled for the first time at the maternity hospital, pre-Cesarean section was performed before the operation.

Of the 50 women who were given intraoperative injections by BMC, 19 (38\%) were consulted at the antenatal period and in the hospital before the operation, and in 31 cases (62\%) consultations were given for the first time at the hospital before the Caesarean section. These results differ from Mishra S.'s findings. [10], who concluded that decisionmaking was better when women were given information in an antenatal clinic. All cases of postplacental BMC administration were performed during a planned C-section operation. Most women (39) were hospitalised for 5 days. However, the other 11 women stayed in the hospital for more than 5 days and for 6-7 days. None of the patients needed to stay longer than 7 days. Haider S. et al. [8] also reported that $94.33 \%$ of women had been in hospital for more than four days. The most frequent complaints at the first observation after 6 weeks were pelvic pains (12\%) followed by vaginal discharge (6\%) and bleeding (4\%). Dikke G.B. [3] also reported similar results. They noted that back / abdominal pain occurred in $14.2 \%$, vaginal discharge in $12.5 \%$ and bleeding in $11.6 \%$. These were the most frequent complaints during the first follow-up visit in 6 weeks. The percentage of women with various complications during the second control visit after 3 months followed almost the same trend as during the first control visit after 6 weeks. Frequent complications at the second follow-up in this study were pelvic pain (12\%), hyperpolymenorrhoea (6\%) and vaginal discharge (4\%). No cases of fever with chills, wound infections or pregnancy were reported. These results are consistent with the findings of Dikke G. B. [3]. At the same time, the number of patients who made complaints decreased significantly.

On the 3 rd control visit after 6 months, pain (8\%) was the most frequent complaint, followed by heavy menstrual bleeding (4\%) and discharge (4\%). In most studies, including the present study, pelvic pain and excessive 
menstrual bleeding were the two most frequent complaints on the third control visit after 6 months. Similarly, pain was the most frequent complaint at a follow-up visit after 6 months in a study conducted by Garcia G. et al [6] (15\%) and Barbosa RM, et al. [4] (35,60\%). While excessive bleeding was the most frequent complaint in the Goldthwaite $L M$, et al [7] (10.41\%), Hubacher D, et al. [9] (14,63\%). In none of the studies was pregnancy with BMC $\mathrm{Cu} \mathrm{T} 380$ A observed. In our study, it was observed that the detection of BMC tendrils increased over time. At the first control visit after 6 weeks they were seen in 72\% (36/50 cases), which increased over time, and at the second control visit after 3 months they were seen in $82 \%$ ( $41 / 50$ cases) and at the end of 6 months they were seen in 90\% (45/50 cases). These results are consistent with the findings of Brunson M. R. et al. [5] who reported no thread in $8 \%$ of the cases. In another Haider $\mathrm{S}$. et al. study, the results are consistent with those of Brunson M. R. et al. [5] who reported no thread in $8 \%$ of cases. In another study by Haider S. et al. [8], the absence of thread was observed in $14.54 \%$ of cases. A comparatively higher percentage of missing threads - about $30 \%$ and $35.45 \%$ - was found in the Dikke G. B. study [3]. Although the number of observations in this study was small, we concluded that the introduction of BMI during Caesarean section is a practical, convenient, safe, effective and acceptable method of contraception to maintain birth spacing in our high birth rate region in rural areas where women have limited access to health services and the likelihood that women will return for postnatal consultations is almost non-existent.

\section{CONCLUSIONS}

1) The intraoperative administration of longacting reversible contraception (LARC) Cu $T 380 \mathrm{~A}$ is a simple, safe, effective contraception with low expulsions and minimal side effects.

2) LARC is the optimal LARC option for lactating and non-lactating women after abdominal delivery.

\section{REFERENCES}

1. An A.V., Pakhomova J.E., Maternal lethality after Caesarean section. Problems of Reproduction No. 3, 2010; pp.83-86.

2. Ayupova F.M., Khamdamova M.T. Clinical aspects of the use of copper-containing intrauterine devices // Uzbekiston Tibbiet Journalist. -2010. № 6. 59-64.

3. Dikke G.B. LARC-methods of first choice in prevention of unwanted pregnancy and repeated abortion // Pharmatek. 2013. №. 12. p. 8-13.

4. Barbosa RM, et al. Differences in the Access to Sterilization between Women Living and Not Living with HIV: Results from the GENIH Study, Brazil PLoS One 2016.

5. Brunson M. R. et al. Postpartum contraception: and effectiveness in a large universal healthcare system //American journal of obstetrics and gynecology. 2017. T. 217. №. 1. Pp. 55. e1-55. e9.

6. Garcia G. et al. Trends and disparities in postpartum sterilization after cesarean section, 2000 through 2008 //Women's Health Issues. 2015. T. 25. №. 6. p. 634-640.

7. Goldthwaite LM, et al. Postpartum intrauterine devices: clinical and programmatic review Am J Obstet Gynecol 2018 - Review.

8. Haider S. et al. A Novel Approach to Postpartum Contraception Provision Combined with Infant Care: A Randomized, Controlled Trial //Women's Health Issues. 2020. T. 30. №. 2. p. 83-92. 
Doi: https://doi.org/10.37547/TAJMSPR/Volume02Issue09-19

9. Hubacher D, et al. Long-acting reversible contraceptive acceptability and unintended pregnancy among women presenting for short-acting methods: a randomized patient preference trial Am J Obstet Gynecol 2017 - Clinical Trial.

10. Mishra S. Evaluation of safety, efficacy, and expulsion of post-placental and intracesarean insertion of intrauterine contraceptive devices (PPIUCD) //The Journal of Obstetrics and Gynecology of India. 2014. T. 64. №. 5. p. 337-343.

11. Starr K. A. et al. Postpartum contraception use by urban/rural status: an analysis of the Michigan pregnancy risk assessment monitoring system data //Women's Health Issues. 2015. T. 25. №. 6. p. 622-627. 